\title{
Psychological Assessment and Quality of Life of patients with Colorectal Cancer at Assiut University Hospital and South Egypt Cancer Institute
}

\author{
Sanaa Mostafa khalifa, Alaa El Din Mohamed Darweesh , Ashraf Zedan Abed Allah, Nadia Ebraheem Sayed \\ Instructor of Psychiatric Nursing Faculty of Nursing - Sohag University \\ Professor of Psychiatry Nursing Faculty of Medicine - Assiut University \\ Professor of Oncology South Egypt Cancer Institute \\ Assistant Professor of Psychiatric Nursing, Faculty of Nursing - Assiut University.
}

\begin{abstract}
Depression, anxiety, stress and poor quality of life are often psychological consequences of living with cancer. This study aimed to assess psychological symptoms and Quality of life among patients with colorectal cancer. A descriptive research design was used to assess patients diagnosed as colorectal cancer. The study was carried out at outpatient clinic of the oncology department at Assiut University Hospital and South Egypt Cancer Institute. Four tools were utilized to measure the variables of the study; the demographic data questionnaire, socioeconomic status scale, Quality of life (QOL) scale and Symptoms Check List (SCL-90-Revised). Results of the study: Females more than males. Most of the patients were living in rural areas, the vast majority of studied group were at middle level of socioeconomic status and have moderate quality of life most of them have cancer colon, (47.6\%) of patients have loss of appetite, (64.2\%) of them have weight loss and didn't have colostomy. (39.7\%) of them have received chemotherapy. A little high percent of patients have somatization and hostility .The study recommend that: liaison psychiatric nurse should be available to deal with psychiatric problems of patients.
\end{abstract}

\section{Key Words: Colorectal Cancer (CRC) - Quality Of Life (QOL)- Symptoms Check List (SCL).}

\section{Introduction}

Colorectal cancer (CRC) includes cancerous growths in the colon, rectum and appendix. Most colorectal cancers arise from adenomatous polyps. These neoplasms are usually benign, but some develop into cancer over time. The occurrence of large bowel cancer is strongly related to age, with $83 \%$ of cases arising in people who are sixty years or older. It is a common form of malignancy in developed countries but occurs much less frequently in the developing world (ONS, 2008; WCISU, 2008; Northern Ireland Cancer Registry, 2008).

Colorectal cancer (CRC) is one of the most common cancers in the world and is the second leading cause of cancer mortality in Western countries. Diet and lifestyle factors are implicated risk factors for the disease. Fruit and vegetable-deficient diet, caloriedense foods, physical inactivity, obesity, and smoking increase the risk for developing CRC (Center et al., 2009). as well as in the United States of America (Jemal et al., 2008). In the Asian population, there is a rapid increase in the trend of CRC incidence (Hyodo et al, 2010) and in Malaysia (National Cancer Registry, 2006).

The emotional adjustment of a patient during treatment depends largely on these secondary side effects (Maté et al., 2003). With the confirmation of diagnosis and the initiation of treatment, both the patient and family are forced to deal with eating disorders, loss of appetite, weight loss, gastrointestinal upset, diarrhea, constipation, altered body image, difficulties in sexual relations, and other related events (Die, 2007).

Patients with colon cancer report serious psychological and emotional morbidities (Fillipovic et al, 2007and Ashbury et al., 2003). Various studies have shown high levels of depression in cancer patients using different methods of assessment (Jadoon, et al., 2010; Pirl, 2004; Mystakidou et al., 2005). It is reported that depression in cancer patients may be caused by diagnosis of cancer, long duration of treatment, side effects of treatment, disruption in life and diminished quality of life(Jadoon, et al., 2010; Mystakidou et al., 2005).

Cancer is one of the diseases with a high psychological impact. This condition can be assessed as stressful because it involves loss and threat (Urquidi et al., 1999). There is no single way to include cancer as part of the subject's life and, much less, is there an order in the adaptive reactions to it. The adjustment to cancer is influenced by society, the patient and the disease itself; therefore, social factors are dynamic (Almanza and Holland, 2000).

Comparisons between cancer survivors and healthy controls provide evidence that cancer survivors face 
ongoing problems related to the cancer diseases including reduction in energy, weight loss, and psychological distress (Schag et a.l, 1994). The possible loss of autonomy, often associated with the feeling of burden, disrupts patient's quality of life (Santos, 1995; Demetri, 2001; Hurter and Bush, 2007). Several studies have assessed the impact of different treatments on patient's quality of life (Schmidt et al, 2005 and Pollack et al., 2006).

Colorectal cancer and its treatment can have an adverse effect on social functioning, including work and productive life; relationships with friends, relatives, and partners; and other social activities and interests. Patients with colorectal cancer, both patients with or without stoma, are troubled by frequent or irregular bowel movements, diarrhea, flatulence, and fatigue, and often have to follow dietary restrictions (Sprangers et al, 1993 and Sprangers et al, 1995).

\section{Significance of the study}

The incidence of colorectal cancer (CRC) has increased in recent years. For this reason, mortality and social impact have become public health problems worldwide. In comparing rates of Egyptian colorectal cancer to the Surveillance Epidemiology and End Results Program (SEER) of the United States, Egypt had higher rates up to age of 3034years, at that point Egyptian rates level out while United States rates increase sharply. With the confirmation of diagnosis and the initiation of treatment, both the patient and family are forced to deal with eating disorders, loss of appetite, weight loss, gastrointestinal upset, diarrhea, constipation, altered body image, difficulties in sexual relations, and other related events. Accordingly, depressive symptoms are also commonly reported in patients suffering from nearly all forms of cancer.

While it is known that psychological factors such as stress and depression adversely affect the quality of life and daily functioning of cancer patients they have also been shown to negatively affect physiological processes leading to ill health. So, exploring psychological symptoms and QOL among patients with CRC could be helpful in designing and implementing psycho educational program for those patients to assist them in coping with disease and its consequence.

\section{Aim of the study}

The study aimed to assess psychological symptoms and Quality of life among patients with colorectal cancer.

\section{Patients and methods Research design}

A descriptive research design was used to assess psychological symptoms and Quality of life among patients with colorectal cancer.

\section{Setting of the study}

The study was conducted at the outpatient clinics of the oncology department at Assiut University Hospital and South Egypt Cancer Institute

\section{Patients (sample)}

The studied sample included (inclusion criteria):

All patients diagnosed as colorectal cancer attending the outpatient clinic for the oncology department and South Egypt Cancer Institute during six months period from $22^{\text {th }}$ January to $22^{\text {th }}$ July 2013 . They included patients received active treatments or those attends the clinic for follow up.

\section{Exclusion criteria}

1-Patients with colorectal cancer have metastasis elsewhere (Metastasis considered a severe form of disease and confounding factor could not be overlooked).

2-Patients with apparent cognitive impairment or general health deterioration (to ensure patient cooperation).

\section{Method}

\section{The following tools were used in the currents} study

1-Demographic and clinical data sheet of the patients

Structured data collection sheet for demographic and clinical characteristics included Patient name, sex, age, occupation, marital status, address ,education level, diagnosis, clinical symptoms e.g eating disturbance (appetite, weight loss), bowel habit disorder ( diarrhea, constipation), type of treatment that the patient received, duration of treatment, colostomy, sexual relation with partner. It was developed by researcher.

\section{2 - Socio economic status data scale}

This scale is an Arabic version designed by AbdEl-Tawab (2004) to assess socioeconomic status of the family and consists of 4 dimensions, which include the following:

- Parent's level of education it included 8 items.

- Parent's occupation it included 2 items.

- Total family monthly income it included 6 items.

- Life style of the family it included 3 items.

The sums of scores give the total score of the variable. The total (raw) score for an individual can be obtained from an equation that depends upon these four variables. Categorization of individuals of a given sample as high, middle, or low socioeconomic class is done as follow: 
1. Calculation of the mean and stander deviation for the total scores of the studied sample.

2. Individuals having scores higher than mean plus one SD are classified as high socioeconomic class.

3. Individuals having scores lower than mean minus one SD are classified as low socioeconomic class.

4. Individuals in between are classified middle class. Accordingly classification of the patients in this study was as follow:

High socioeconomic class scores are more than 123.7

Middle socioeconomic class scores range between 53 and 123.7 Low socioeconomic class scores are less than 53

3-Quality of life scale (QOL) lehman et al., (1986): It's was constructed by Lehman et al (1986) to assess QOL of psychiatric patient. It consists of 57 items divided into six domains or subscales modified and cited in Abed El Wahab et al, (2002).

- First subscale, comprising 12 items covering the physical health.

- The second subscale consisted of 8 items reflecting self care.

- The third sub scale included 14 items, representing patient's emotional status.

- The fourth one consisted of 13 items related to personal \&social relationship.

- The fifth subscale included 5 items which assess the patient's ability to make decision, to work, to carry out the work duties and ability to taking responsibility.

- The last subscale consisted of 5 items used to collect data about spiritual concerns and personal beliefs.

Reponses were measured on three points likert scale, ranging from 0 to 2 The total score was 114 points. Individual obtained a score less than 57 points were considered to have a low quality of life, while those who scored between 57: 85 points were considered to have a moderate quality of life, and finally, those who scored more than 85 points were considered to have a high quality of life. In this study alpha cronbach's of the Quality Of Life was found to be 0.75 .

4-The symptom checklist (SCL-90-Revised) (Elbehairy, 2004) (Appendix VI)

Descriptive profile

It comprised of 90-items self-report symptom inventory developed by clinical psychometric research. It was designed primarily to reflect the psychological symptoms pattern of psychiatric and medical patients. A preliminary version of the scale was introduced by Derogatis and his colleagues (Derogatis et al., 1973) and based on early clinical experiences. Psychometric analysis was modified and validated in the revised (R) form (Derogatis et al., 1976). Translated by Elbehairy, 2004.

Each item of this scale " 90 " is rated on a 5-point scale of distress (0-4), ranging from non-at-all at one pole to "extremely " at the other pole The " 90 " is scored and interpreted in terms of 9 primary symptom dimensions and 3 global indices of distress that are labeled:

1.Somatization,

2.Obsessive compulsive,

3.Interpersonal sensitivity.

4.Depression.

5.Anxiety, 6.Hostility, 7.Phobic anxiety, 8.Paranoid ideation, 9.Psychoticism.

The previous question were translated into Arabic language this translation was revised by five expertise; three assistant professor in psychiatric nursing and two professor of psychiatric medicine .A back translation into English language compared with the original sentence to ensure content validity.

\section{Procedure}

1-After appraisal of the protocol of the study for ethical and scientific committee an official letter from the faculty of Nursing Assiut University directed to the Head of oncology department at Assiut University Hospital and Dean of South Egypt Cancer Institute in order to get permission to conduct the study.

2-A descriptive research used to conduct study.

3-The aim of the study explained to the patients before starting data collection.

4-Oral consent was taken from all patients who reassured about the confidentiality of the obtained information to avoid misunderstanding.

5- Application of the tools of the study it took about from 80 minutes to 90 minutes.

6-Each participant interviewed and assessed individually.

7- The data collection took a period of six months, from 22 January to 22 July 2013, 3 days per week during the morning from 9 am to $2 \mathrm{pm}$.

\section{Pilot Study}

A pilot study was carried to test the clarity of the tool, and to estimate the time required to fill out the form. It was be carried out on 15 patients and excluded from the actual study sample.

Statistical Analysis

The data were computerized and verified using the SPSS (Statistical Package for Social Science) version 16.00 to perform tabulation and statistical analysis. Qualitative variables were described in frequency and percentages, while quantitative variables were described by mean and standard deviation. Analysis of collected data was done through the use of several 
statistical tests as: chi-square test $\left(\mathrm{x}^{2}\right)$ was used to analyze qualitative variables and $t$ test for quantitative variables. Statistical significance was considered at $\mathrm{P}$ - value $<0.05$.

Significance was considered at $\mathrm{P}$ - value $<0.05$.

\section{Ethical considerations}

1-Risk-benefit assessment: There is no risk during application of the research.

2-Confidentiality was maintained during the research.

3-Informed oral consent was taken from all patients for their approval to participate in this study.

\section{Results}

Results of the present study showed that:-

Table (1): Demographic characteristics of the studied patients with colorectal cancer $($ No=151).

\begin{tabular}{|c|c|c|c|c|c|c|}
\hline Demographic characteristic & \multicolumn{2}{|c|}{ Male $(\mathrm{N}=54)$} & \multicolumn{2}{|c|}{ Female $(\mathrm{N}=97)$} & T-test & P-value \\
\hline Age of total group $(45.31 \pm 13.45)$ & \multicolumn{2}{|c|}{$\begin{array}{c}\text { Mean } \pm \text { SD } \\
44.94 \pm 14.25\end{array}$} & \multicolumn{2}{|c|}{$\begin{array}{c}\text { Mean } \pm \text { SD } \\
45.51 \pm 13.05\end{array}$} & 41.83 & .000 \\
\hline \multirow{2}{*}{\begin{tabular}{ll}
\multicolumn{2}{l}{ Residence } \\
Rural & $($ no=115) $76.16 \%$ \\
Urban & $($ no=36) $23.84 \%$ \\
\end{tabular}} & No & $\%$ & No & $\%$ & $\mathbf{X}^{2}$ & P-value \\
\hline & $\begin{array}{l}43 \\
11 \\
\end{array}$ & $\begin{array}{l}79.6 \\
20.4\end{array}$ & $\begin{array}{l}72 \\
25\end{array}$ & $\begin{array}{l}74.2 \\
25.8\end{array}$ & .558 & .455 \\
\hline $\begin{array}{l}\text { Level of education } \\
\text { Illiterate or read and write }(\text { no }=119) 78.8 \% \\
\text { Technical school }(\text { no }=20) 13.24 \% \\
\text { University education }(n o=12) 7.94 \%\end{array}$ & $\begin{array}{c}38 \\
9 \\
7\end{array}$ & $\begin{array}{l}70.4 \\
16.7 \\
13.0 \\
\end{array}$ & $\begin{array}{c}81 \\
11 \\
5 \\
\end{array}$ & $\begin{array}{c}83.54 \\
11.3 \\
5.2 \\
\end{array}$ & 7.833 & .098 \\
\hline $\begin{array}{l}\text { Occupation } \\
\text { Not work }(\text { no }=116) 76.8 \% \\
\text { Not skill worker }(\text { no }=20) 13.24 \% \\
\text { Employee }(\text { no }=12) 7.94 \% \\
\text { Student }(\text { no }=3) 1.98 \%\end{array}$ & $\begin{array}{l}23 \\
19 \\
9 \\
3\end{array}$ & $\begin{array}{c}42.6 \\
35.2 \\
16.7 \\
5.6\end{array}$ & $\begin{array}{c}93 \\
1 \\
3 \\
0\end{array}$ & $\begin{array}{c}95.9 \\
1.0 \\
3.1 \\
0\end{array}$ & 57.069 & .000 \\
\hline 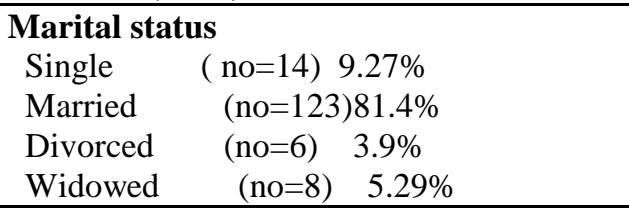 & $\begin{array}{c}8 \\
46 \\
0 \\
0\end{array}$ & $\begin{array}{c}14.8 \\
85.2 \\
0 \\
0\end{array}$ & $\begin{array}{c}6 \\
77 \\
6 \\
8 \\
\end{array}$ & $\begin{array}{c}6.2 \\
79.4 \\
6.2 \\
8.2 \\
\end{array}$ & 10.723 & .013 \\
\hline $\begin{array}{l}\text { Socioeconomic status level } \\
\text { Low } \quad(\text { no }=4) 2.64 \% \\
\text { Moderate }(\mathrm{no}=122) 80.8 \% \\
\text { High } \quad(\mathrm{no}=25) 16.55 \%\end{array}$ & $\begin{array}{c}2 \\
40 \\
12\end{array}$ & $\begin{array}{c}3.7 \\
74.1 \\
22.2\end{array}$ & $\begin{array}{c}2 \\
82 \\
13\end{array}$ & $\begin{array}{l}2.1 \\
84.5 \\
13.4\end{array}$ & 2.453 & .293 \\
\hline
\end{tabular}


Table (2): Frequency of symptoms related to colorectal cancer among the studied patients.

\begin{tabular}{|c|c|c|c|c|c|c|c|c|}
\hline \multirow{3}{*}{ Clinical picture } & \multicolumn{8}{|c|}{ diagnosis } \\
\hline & \multicolumn{2}{|c|}{ Colon $(\mathrm{N}=94)$} & \multicolumn{2}{|c|}{$\operatorname{Rectum}(\mathrm{N}=54)$} & \multicolumn{2}{|c|}{ Anal (N=3) } & \multirow[b]{2}{*}{$\mathbf{X} 2$} & \multirow{2}{*}{ P-value } \\
\hline & No & $\%$ & No & $\%$ & No & $\%$ & & \\
\hline $\begin{array}{l}\text { Bowel habit } \\
\text { Not change } \quad(\text { no= }=87) 57.61 \% \\
\text { Diarrhea } \quad(n o=37) 24.50 \% \\
\text { Constipation }(n o=27) 17.88 \% \\
\end{array}$ & $\begin{array}{l}57 \\
20 \\
17 \\
\end{array}$ & $\begin{array}{l}60.6 \\
21.3 \\
18.1 \\
\end{array}$ & $\begin{array}{c}29 \\
16 \\
9 \\
\end{array}$ & $\begin{array}{l}53.7 \\
29.6 \\
16.7 \\
\end{array}$ & $\begin{array}{l}1 \\
1 \\
1 \\
\end{array}$ & $\begin{array}{l}33.3 \\
33.3 \\
33.3 \\
\end{array}$ & 2.121 & .714 \\
\hline $\begin{array}{l}\text { Appetite } \\
\text { Not change } \quad(\text { no= }=75) 49.66 \% \\
\text { Decreased appetite }(\text { no }=72) 47.68 \% \\
\text { Increased appetite }(\text { no }=4) 2.64 \% \\
\end{array}$ & $\begin{array}{c}47 \\
45 \\
2 \\
\end{array}$ & $\begin{array}{c}50.0 \\
47.9 \\
2.1 \\
\end{array}$ & $\begin{array}{c}27 \\
25 \\
2 \\
\end{array}$ & $\begin{array}{c}50.0 \\
46.3 \\
3.7 \\
\end{array}$ & $\begin{array}{l}1 \\
2 \\
0\end{array}$ & $\begin{array}{c}33.3 \\
66.7 \\
0 \\
\end{array}$ & .816 & .936 \\
\hline $\begin{array}{l}\text { Weight loss } \\
\text { Yes } \quad(\text { no=97) } 64.23 \% \\
\text { No } \quad(\text { no=54) } 35.76 \% \\
\end{array}$ & $\begin{array}{l}57 \\
37 \\
\end{array}$ & $\begin{array}{l}60.6 \\
39.4 \\
\end{array}$ & $\begin{array}{l}37 \\
17 \\
\end{array}$ & $\begin{array}{l}68.5 \\
31.5 \\
\end{array}$ & $\begin{array}{l}3 \\
0 \\
\end{array}$ & $\begin{array}{c}100.0 \\
0 \\
\end{array}$ & 2.631 & .268 \\
\hline $\begin{array}{l}\text { Have colostomy } \\
\text { Yes }(\text { no=37) } 24.50 \% \\
\text { No }(\text { no }=114) 75.49 \%\end{array}$ & $\begin{array}{l}15 \\
79 \\
\end{array}$ & $\begin{array}{l}16.0 \\
84.0 \\
\end{array}$ & $\begin{array}{l}21 \\
33 \\
\end{array}$ & $\begin{array}{l}38.9 \\
61.1 \\
\end{array}$ & $\begin{array}{l}1 \\
2 \\
\end{array}$ & $\begin{array}{l}33.3 \\
66.7 \\
\end{array}$ & 9.878 & .007 \\
\hline $\begin{array}{l}\text { Line of treatment } \\
\text { Non specific treatment }(\mathrm{no}=80) 52.98 \% \\
\text { Chemotherapy }(\mathrm{no}=60) 39.73 \% \\
\text { Radiotherapy }(\mathrm{no}=5) 3.31 \% \\
\text { Combined medication }(\mathrm{no}=6) 3.97 \%\end{array}$ & $\begin{array}{c}49 \\
38 \\
4 \\
3\end{array}$ & $\begin{array}{l}52.1 \\
40.4 \\
4.3 \\
3.2 \\
\end{array}$ & $\begin{array}{c}31 \\
20 \\
0 \\
3 \\
\end{array}$ & $\begin{array}{c}57.4 \\
37.0 \\
0 \\
5.6 \\
\end{array}$ & $\begin{array}{l}0 \\
2 \\
1 \\
0\end{array}$ & $\begin{array}{c}0 \\
66.7 \\
33.3 \\
0\end{array}$ & 13.27 & .039 \\
\hline
\end{tabular}

Table (3): Frequency of different levels of quality of life (QOL) of colorectal cancer patients.

\begin{tabular}{|c|c|c|c|c|c|c|c|c|}
\hline \multirow[t]{2}{*}{ Demographic characteristics } & \multicolumn{2}{|c|}{$\begin{array}{c}\text { Low QOL } \\
(\mathrm{No}=8)\end{array}$} & \multicolumn{2}{|c|}{$\begin{array}{c}\text { Moderate } \\
\text { QOL }(\text { No=99) }\end{array}$} & \multicolumn{2}{|c|}{$\begin{array}{c}\text { High QOL } \\
(\text { No=44) }\end{array}$} & \multirow[t]{2}{*}{$\mathrm{X} 2$} & \multirow[t]{2}{*}{ P-value } \\
\hline & No & $\%$ & No & $\%$ & No & $\%$ & & \\
\hline $\begin{array}{ll}\text { Sex } & \\
\text { Male } & (\text { no=54) } 35.76 \% \\
\text { Female } & (\text { no=95) } 64.91 \%\end{array}$ & $\begin{array}{l}3 \\
5\end{array}$ & $\begin{array}{l}37.5 \\
62.5\end{array}$ & $\begin{array}{l}33 \\
66\end{array}$ & $\begin{array}{l}33.3 \\
66.7\end{array}$ & $\begin{array}{l}18 \\
26\end{array}$ & $\begin{array}{l}40.9 \\
59.1\end{array}$ & .772 & .680 \\
\hline \begin{tabular}{lcl}
\multicolumn{2}{l}{ Marital status } \\
Single & $($ no=14) & $9.27 \%$ \\
Married & $($ no=123) & $81.4 \%$ \\
Divorced & $($ no $=6)$ & $3.9 \%$ \\
Widowed & $($ no $=8)$ & $5.29 \%$
\end{tabular} & $\begin{array}{l}2 \\
6 \\
0 \\
0\end{array}$ & $\begin{array}{c}25.0 \\
75.0 \\
0 \\
0\end{array}$ & $\begin{array}{c}6 \\
85 \\
4 \\
4\end{array}$ & $\begin{array}{c}6.1 \\
\mathbf{8 5 . 9} \\
4.0 \\
4.0\end{array}$ & $\begin{array}{c}6 \\
32 \\
2 \\
4\end{array}$ & $\begin{array}{l}13.6 \\
72.7 \\
4.5 \\
9.1\end{array}$ & 7.097 & .312 \\
\hline $\begin{array}{ll}\text { Residence } & \\
\text { Rural } & (\mathrm{no}=115) 76.16 \% \\
\text { Urban } & (\mathrm{no}=36) 23.84 \%\end{array}$ & $\begin{array}{l}5 \\
3\end{array}$ & $\begin{array}{l}62.5 \\
37.5\end{array}$ & $\begin{array}{l}77 \\
22\end{array}$ & $\begin{array}{c}\mathbf{7 7 . 8 \%} \\
22.2\end{array}$ & $\begin{array}{l}33 \\
11\end{array}$ & $\begin{array}{l}75.0 \\
25.0\end{array}$ & .997 & .607 \\
\hline $\begin{array}{l}\text { Level of education } \\
\text { Illiterate or read and write (no=119) } \\
78.8 \% \\
\text { Technical school }(\mathrm{no}=20) 13.24 \% \\
\text { University education }(\mathrm{no}=12) 7.94 \% \\
\end{array}$ & $\begin{array}{l}6 \\
1 \\
1 \\
\end{array}$ & $\begin{array}{l}75.0 \\
12.5 \\
12.5 \\
\end{array}$ & $\begin{array}{c}79 \\
15 \\
5 \\
\end{array}$ & $\begin{array}{c}79.7 \\
15.2 \\
5.1 \\
\end{array}$ & $\begin{array}{c}34 \\
4 \\
6 \\
\end{array}$ & $\begin{array}{c}77.2 \\
9.1 \\
13.6 \\
\end{array}$ & 5.307 & .724 \\
\hline $\begin{array}{l}\text { Occupation } \\
\text { Not work (no=116) } 76.8 \% \\
\text { Not skill worker }(\text { no }=20) 13.24 \% \\
\text { Employee }(\text { no=12) } 7.94 \% \\
\text { Student (no=3) } 1.98 \%\end{array}$ & $\begin{array}{l}6 \\
0 \\
1 \\
1\end{array}$ & $\begin{array}{c}75.0 \\
0 \\
12.5 \\
12.5\end{array}$ & $\begin{array}{c}81 \\
12 \\
5 \\
1\end{array}$ & $\begin{array}{c}\mathbf{8 1 . 8} \\
12.2 \\
5.1 \\
1.0\end{array}$ & $\begin{array}{c}29 \\
8 \\
6 \\
1\end{array}$ & $\begin{array}{c}65.9 \\
18.2 \\
13.6 \\
2.3\end{array}$ & 11.32 & .184 \\
\hline $\begin{array}{l}\text { Socioeconomic status level } \\
\text { Low } \quad(\text { no }=4) 2.64 \% \\
\text { Moderate }(\text { no }=122) 80.8 \% \\
\text { High } \quad(\text { no }=25) 16.55 \%\end{array}$ & $\begin{array}{l}0 \\
8 \\
0\end{array}$ & $\begin{array}{c}0 \\
100 \\
0\end{array}$ & $\begin{array}{c}4 \\
77 \\
18\end{array}$ & $\begin{array}{c}4.0 \\
\mathbf{7 7 . 8} \\
18.2\end{array}$ & $\begin{array}{c}0 \\
37 \\
7\end{array}$ & $\begin{array}{c}0 \\
84.1 \\
15.9\end{array}$ & 1.823 & 0.768 \\
\hline
\end{tabular}


Table (4): Relationship between clinical variables of patients with colorectal cancer and their levels of quality of life.

\begin{tabular}{|c|c|c|c|c|c|c|c|c|}
\hline \multirow[t]{2}{*}{ Clinical variable } & \multicolumn{2}{|c|}{$\begin{array}{c}\text { Low QOL } \\
(\text { No=8) }\end{array}$} & \multicolumn{2}{|c|}{$\begin{array}{c}\text { Moderate QOL } \\
(\text { No=99) }\end{array}$} & \multicolumn{2}{|c|}{$\begin{array}{c}\text { High QOL } \\
(\text { No }=44)\end{array}$} & \multirow[t]{2}{*}{$\mathrm{X} 2$} & \multirow[t]{2}{*}{ P-value } \\
\hline & No & $\%$ & No & $\%$ & No & $\%$ & & \\
\hline $\begin{array}{l}\text { Diagnosis } \\
\text { Cancer colon }(\mathrm{n}=94) 62.25 \% \\
\text { Cancer rectum }(\mathrm{n}=54) 35.76 \% \\
\text { Cancer anal }(\mathrm{no}=3) 1.98 \%\end{array}$ & $\begin{array}{l}4 \\
4 \\
0\end{array}$ & $\begin{array}{c}50.0 \\
50.0 \\
0 \\
\end{array}$ & $\begin{array}{c}62 \\
35 \\
2\end{array}$ & $\begin{array}{c}\mathbf{6 2 . 6} \\
35.4 \\
2.0 \\
\end{array}$ & $\begin{array}{c}28 \\
15 \\
1\end{array}$ & $\begin{array}{c}63.6 \\
34.1 \\
2.3 \\
\end{array}$ & .879 & .928 \\
\hline $\begin{array}{l}\text { Sexual state } \\
\text { Not change (no=20) } 13.24 \% \\
\text { less (no=95) } 62.91 \%\end{array}$ & $\begin{array}{l}0 \\
5\end{array}$ & $\begin{array}{c}0 \\
62.5\end{array}$ & $\begin{array}{l}10 \\
66\end{array}$ & $\begin{array}{l}10.1 \\
\mathbf{6 8 . 7}\end{array}$ & $\begin{array}{l}10 \\
26\end{array}$ & $\begin{array}{c}22.7 \\
50\end{array}$ & 11.89 & .064 \\
\hline 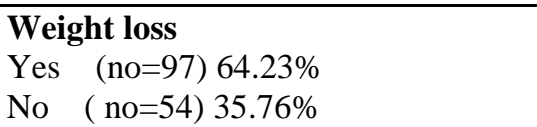 & $\begin{array}{l}6 \\
2\end{array}$ & $\begin{array}{l}75.0 \\
25.0\end{array}$ & $\begin{array}{l}69 \\
30\end{array}$ & $\begin{array}{r}\mathbf{6 8 . 7} \\
31.3\end{array}$ & $\begin{array}{l}22 \\
22\end{array}$ & $\begin{array}{l}50.0 \\
50.0\end{array}$ & 5.570 & .062 \\
\hline $\begin{array}{l}\text { Bowel habit } \\
\text { Not change (no=87) } 57.61 \% \\
\text { Diarrhea (no=37) } 24.50 \% \\
\text { Constipation }(\mathrm{no}=27) 17.88 \% \\
\end{array}$ & $\begin{array}{l}3 \\
2 \\
3 \\
\end{array}$ & $\begin{array}{l}37.5 \\
25.0 \\
37.5\end{array}$ & $\begin{array}{l}52 \\
27 \\
20 \\
\end{array}$ & $\begin{array}{l}\mathbf{5 2 . 5} \\
27.3 \\
20.2\end{array}$ & $\begin{array}{l}32 \\
8 \\
4 \\
\end{array}$ & $\begin{array}{c}72.7 \\
18.2 \\
9.1 \\
\end{array}$ & 7.701 & .103 \\
\hline $\begin{array}{l}\text { Appetite } \\
\text { Not change (no=75) } 49.66 \% \\
\text { Less }(\text { no=72) } 74.68 \% \\
\text { More }(\mathrm{no}=4) 2.64 \\
\end{array}$ & $\begin{array}{l}1 \\
6 \\
1\end{array}$ & $\begin{array}{l}12.5 \\
75.0 \\
12.5\end{array}$ & $\begin{array}{l}47 \\
50 \\
2 \\
\end{array}$ & $\begin{array}{l}47.5 \\
\mathbf{5 0 . 5} \\
2.0 \\
\end{array}$ & $\begin{array}{c}27 \\
16 \\
1 \\
\end{array}$ & $\begin{array}{c}61.4 \\
36.4 \\
2.3 \\
\end{array}$ & 9.234 & .056 \\
\hline $\begin{array}{l}\text { Have colostomy } \\
\text { Yes }(\mathrm{no}=37) 24.50 \% \\
\text { No }(\mathrm{no}=114) 75.49 \% \\
\end{array}$ & $\begin{array}{l}2 \\
6 \\
\end{array}$ & $\begin{array}{l}25.0 \\
75.0 \\
\end{array}$ & $\begin{array}{l}27 \\
72 \\
\end{array}$ & $\begin{array}{l}27.3 \\
\mathbf{7 2 . 7}\end{array}$ & $\begin{array}{c}8 \\
36 \\
\end{array}$ & $\begin{array}{l}18.2 \\
81.8 \\
\end{array}$ & 1.362 & .506 \\
\hline $\begin{array}{l}\text { Line of treatment } \\
\text { Non specific treatment }(\mathrm{no}=80) \\
52.98 \% \\
\text { Chemotherapy(no=60) } 39.73 \% \\
\text { Radiotherapy(no=5) } 3.31 \% \\
\text { Combined medication }(\mathrm{no}=6) 3.97 \%\end{array}$ & $\begin{array}{l}6 \\
1 \\
1 \\
0\end{array}$ & $\begin{array}{c}75.0 \\
12.5 \\
12.5 \\
0\end{array}$ & $\begin{array}{c}52 \\
37 \\
4 \\
6\end{array}$ & $\begin{array}{c}\mathbf{5 2 . 5} \\
37.4 \\
4.0 \\
6.1\end{array}$ & $\begin{array}{c}22 \\
22 \\
0 \\
0\end{array}$ & $\begin{array}{c}50.0 \\
50.0 \\
0 \\
0\end{array}$ & 10.41 & .108 \\
\hline
\end{tabular}

Table (5): Frequency of male and female patients who need psychiatric assistance at different dimensions of SCL- 90-R (score $\geq 60$ ).

\begin{tabular}{|l|c|c|c|c|c|c|}
\hline \multirow{2}{*}{ Dimensions of SCL- 90- R } & \multicolumn{9}{|c|}{ sex } \\
\cline { 2 - 6 } & \multicolumn{2}{|c|}{ Male (No=54) } & \multicolumn{2}{c|}{ Female (No=97) } & \multirow{2}{*}{$\mathbf{X}^{\mathbf{2}}$} & \multirow{2}{*}{ P-value } \\
\cline { 2 - 6 } & $\mathbf{N o}$ & $\mathbf{\%}$ & $\mathbf{N o}$ & $\mathbf{\%}$ & & \\
\hline Somatization (no=49) 32.45\% & 17 & 34.7 & 32 & 65.3 & .036 & .850 \\
\hline Obsession (no=3) 1.98\% & 2 & 66.7 & 1 & 33.3 & 1.273 & .259 \\
\hline Sensitivity (no=4) 2.64\% & 1 & 25.0 & 3 & 75.0 & .207 & .649 \\
\hline Depression (no=13) 8.60\% & 3 & 23.1 & 10 & 76.9 & .996 & .318 \\
\hline Anxiety (no=14) 9.27\% & 1 & 7.1 & 13 & 92.9 & 5.501 & .019 \\
\hline Hostility (no=42) 27.81\% & 9 & 21.4 & 33 & 78.6 & 5.203 & .023 \\
\hline Phobia (no=9) 5.96\% & 3 & 33.3 & 6 & 66.7 & .025 & .875 \\
\hline Paranoia (no=8) 5.29\% & 2 & 25.0 & 6 & 75.0 & .426 & .514 \\
\hline Psychoticism (no=6) 3.97\% & $\mathbf{1}$ & $\mathbf{1 6 . 7}$ & $\mathbf{5}$ & $\mathbf{8 3 . 3}$ & $\mathbf{. 9 9 2}$ & $\mathbf{. 3 1 9}$ \\
\hline
\end{tabular}


Table (6): Relationship between levels of QOL and different dimensions of SCL- 90-R in patients who need psychiatric assistance (score $\geq 60$ ).

\begin{tabular}{|c|c|c|c|c|c|c|c|c|}
\hline \multirow{3}{*}{ Dimensions of SCL- 90- R } & \multicolumn{8}{|c|}{ QOL } \\
\hline & \multicolumn{2}{|c|}{$\begin{array}{c}\text { Low QOL } \\
(\text { No=8) }\end{array}$} & \multicolumn{2}{|c|}{ Moderate QOL (No=99) } & \multicolumn{2}{|c|}{$\begin{array}{c}\text { High QOL } \\
(\text { No }=44)\end{array}$} & \multirow[t]{2}{*}{$\mathbf{X}^{2}$} & \multirow[t]{2}{*}{ P-value } \\
\hline & No & $\%$ & No & $\%$ & No & $\%$ & & \\
\hline Somatization $(\mathrm{no}=49) 32.45 \%$ & 7 & 14.4 & 39 & 79.6 & 3 & 6.1 & 26.42 & .000 \\
\hline Obsession $\quad($ no $=3) 1.98 \%$ & 2 & 66.7 & 1 & 33.3 & 0 & 0 & 23.13 & .000 \\
\hline Sensitivity $(\mathrm{no}=4) 2.64 \%$ & 2 & 50 & 2 & 50 & 0 & 0 & 16.84 & .000 \\
\hline Depression $\quad(n o=13) 8.60 \%$ & 5 & 38.5 & 8 & 61.5 & 0 & 0 & 33.70 & .000 \\
\hline$($ no=14) $9.27 \%$ & 5 & 35.7 & 9 & 64.3 & 0 & 0 & 31.44 & .000 \\
\hline Hostility $\quad($ no=42) $27.81 \%$ & 6 & 14.3 & 35 & 83.3 & 1 & 2.4 & 25.97 & .000 \\
\hline$(n o=9) 5.96 \%$ & 2 & 22.2 & 7 & 77.8 & 0 & 0 & 8.18 & .017 \\
\hline$(n o=8) 5.29 \%$ & 4 & 50 & 3 & 37.5 & 1 & 12.5 & 33.67 & .000 \\
\hline Psychoticism $($ no=6) $3.97 \%$ & 3 & 50 & 3 & 50 & 0 & 0 & 25.61 & .000 \\
\hline
\end{tabular}

Table (7): Frequency of the patients who need psychiatric assistance as regard different dimensions of SCL- 90-R according to their lines of treatments.

\begin{tabular}{|c|c|c|c|c|c|c|c|c|c|c|}
\hline \multirow{3}{*}{ Dimensions of SCL- 90- R } & \multicolumn{10}{|c|}{ Treatment } \\
\hline & \multicolumn{2}{|c|}{$\begin{array}{c}\text { Nonspecific } \\
\text { treatment } \\
(\mathrm{No}=\mathbf{8 0})\end{array}$} & \multicolumn{2}{|c|}{$\begin{array}{c}\text { Chemotherapy } \\
\text { treatment } \\
(\mathrm{No}=60)\end{array}$} & \multicolumn{2}{|c|}{$\begin{array}{c}\text { Radiotherapy } \\
\text { treatment } \\
(\mathrm{No}=5)\end{array}$} & \multicolumn{2}{|c|}{$\begin{array}{r}\text { Combined } \\
\text { medication } \\
(\mathrm{No}=6)\end{array}$} & \multirow[t]{2}{*}{$\mathbf{X} 2$} & \multirow[t]{2}{*}{ P-value } \\
\hline & No & $\%$ & No & $\%$ & No & $\%$ & No & $\%$ & & \\
\hline Somatization $(n o=49) \quad 32.45 \%$ & 27 & 55.1 & 13 & 26.5 & 4 & 8.2 & 5 & 10.2 & 15.489 & .001 \\
\hline Obsession $\quad(n o=3) 1.98 \%$ & 1 & 66.7 & 2 & 33.3 & 0 & 0 & 0 & 0 & 1.005 & .800 \\
\hline Sensitivity $($ no=4) $2.64 \%$ & 1 & 25.0 & 3 & 75.0 & 0 & 0 & 0 & 0 & 2.192 & .533 \\
\hline Depression $(\mathrm{no}=13) 8.60 \%$ & 8 & 61.5 & 2 & 15.4 & 2 & 15.4 & 1 & 7.7 & 9.076 & .028 \\
\hline Anxiety $\quad($ no=14) $9.27 \%$ & 11 & 87.6 & 1 & 7.1 & 2 & 14.3 & 0 & 0 & 12.258 & .007 \\
\hline Hostility $\quad($ no=42) $27.81 \%$ & 20 & 7.1 & 13 & 66.7 & 5 & 9.5 & 4 & 16.7 & 18.932 & .000 \\
\hline Phobia $\quad($ no=9) $5.96 \%$ & 5 & 55.6 & 3 & 33.3 & 1 & 11.1 & 0 & 0 & 2.249 & .522 \\
\hline Paranoia $\quad(n o=8) 5.29 \%$ & 6 & 75.0 & 1 & 12.5 & 0 & 0 & 1 & 12.5 & 4.175 & .243 \\
\hline Psychoticism $(n o=6) 3.97 \%$ & 4 & 66.7 & 1 & 16.7 & 1 & 16.7 & 0 & 0 & 4.672 & .197 \\
\hline
\end{tabular}

Table (8): Frequency of patients who need psychiatric assistance at different dimensions of SCL- 90-R according to presence or absence of colostomy.

\begin{tabular}{|c|c|c|c|c|c|c|}
\hline \multirow{3}{*}{ Dimensions of SCL- 90- R } & \multicolumn{6}{|c|}{ Colostomy } \\
\hline & \multicolumn{2}{|c|}{ Yes $(\mathrm{No}=37)$} & \multicolumn{2}{|c|}{ NO $(\mathrm{No}=114)$} & \multirow[t]{2}{*}{$\overline{X^{2}}$} & \multirow{2}{*}{$\begin{array}{c}P- \\
\text { value }\end{array}$} \\
\hline & No & $\%$ & No & $\%$ & & \\
\hline Somatization $(n o=49) 32.45 \%$ & 13 & 26.5 & 36 & 73.5 & .161 & .688 \\
\hline $\begin{array}{lll}\text { Obsession } & (\text { no }=3) & 1.98 \%\end{array}$ & 1 & 33.3 & 2 & 66.7 & .129 & .719 \\
\hline Sensitivity $(\mathrm{no}=4) 2.64 \%$ & 2 & 50 & 2 & 50 & 1.444 & .230 \\
\hline Depression $\quad(n o=13) 8.60 \%$ & 4 & 30.8 & 9 & 69.2 & .302 & .583 \\
\hline Anxiety $\quad(n o=14) 9.27 \%$ & 3 & 21.4 & 11 & 78.6 & .079 & .779 \\
\hline Hostility $\quad($ no=42) $27.81 \%$ & 11 & 26.2 & 31 & 73.8 & .090 & .765 \\
\hline Phobia $\quad(n o=9) 5.96 \%$ & 4 & 44.4 & 5 & 55.6 & 2.057 & .151 \\
\hline Paranoia $\quad($ no=8) $5.29 \%$ & 6 & 75.0 & 2 & 25.0 & 11.644 & .001 \\
\hline $\begin{array}{ll}\text { Psychoticism } & (\mathrm{no}=6) \quad 3.97 \% \\
\end{array}$ & 1 & 16.7 & 5 & 83.3 & .207 & .649 \\
\hline
\end{tabular}


It is known that psychological factors such as stress and depression adversely affect the quality of life and daily functioning of cancer patients they have also been shown to negatively impact physiological processes leading to ill health.

Table (1) : illustrate that mean age of the studied patients was $(45.31 \pm 13.45), 64.2 \%$ of them were females , $35.7 \%$ of them were males ,higher percent of them live in rural area , $78.8 \%$ of them illiterate or read and write $76.8 \%$ of them did not worked, $81.4 \%$ of them were married and $80.8 \%$ at middle socioeconomic status, with no significance differences among these groups. Females were significantly older than male. Significantly higher percent of male were married $(85.2 \%)$ and significantly higher percent of female $(95.9 \%)$ were not worked.

Table (2) : shows the frequency of symptoms among the studied colorectal cancer of patients, it was found that, $60.6 \%$ of cancer colon patients have no change in gastric state, $50 \%$ of colon and rectum cancer patients haven't any change in appetite, $68.5 \%$ of cancer rectum patients have weight loss, about $84.0 \%$ of cancer colon patients have not colostomy and lastly $66.7 \%$ of cancer anal patients receive nonspecific treatment. There are significant differences among patients as regard presence of colostomy and line of treatments.

Table (3) : illustrates frequency of different levels of quality of life (QOL) of colorectal cancer patients. Higher percent of patients have moderate level of quality of life $(65.56 \%)$ of them $66.7 \%$ were females , $85.9 \%$ were married ,77.8\% of them live in rural area , $79.7 \%$ were illiterate or read and write, $81.8 \%$ of them did not work and lastly $77.8 \%$ of them were in middle socioeconomic level. On the other hand, small percent of patients have low QOL (5.2\%).

As regards patients who have moderate QOL as they represent the main group of patients, $62.6 \%$ of them have cancer colon, $68.7 \%$ of them have less than usual sexual relation, $68.7 \%$ of them have weight loss, $52.5 \%$ of them have no change in gastric state, $50.5 \%$ of them have less change in the appetite, $72.7 \%$ of them haven't colostomy and lastly, $52.5 \%$ of them received nonspecific treatment (table 4).

Table (5) : shows anxiety and hostility are significantly higher prevalent in female have than males ( $\mathrm{p}=.01, .02$ respectively). However, other SCL dimensions are non-significant higher in female.

Table (6) : shows that significantly higher percentages of colorectal cancer patients who have somatization $(79.6 \%)$, depression $(61.5 \%)$, anxiety $(64.3 \%)$, hostility $(83.3 \%)$ and phobia $(77.8 \%)$, have moderate level of quality of life. Also significantly higher percentages of colorectal cancer patients who have obsession $(66.7 \%)$, interpersonal sensitivity
(50\%), paranoia $(50 \%)$ and psychosis $(50 \%)$ have low level of quality of life.

Table (7) : shows that significant higher percentage of patients who need psychiatric help as regard somatization (55.1\%), depression (61.5\%) and anxiety $(87.6 \%)$ receive nonspecific treatment.

Table (8) : shows only a high significant percentages among patients who have colostomy need psychiatric assistance as regard Paranoia dimension(75.0\%) of SCL- 90-R ( $\mathrm{p}=.001)$. Otherwise there is no significant difference among both group.

\section{Discussion}

In the current study females represent about two thirds of the sample which is higher than male. Also female were significantly older than male. This higher prevalence of female was reported by Salvador et al., (2011) they found that female represented more than half of the sample of their studies and Landsbergen et al., (2011) they found that females more affected than males. However, the current study is contraindicated with other studies that carried out by Maria et al., (2012) found male $59.5 \%$ and female $40.5 \%$, Ahmetet al., (2008) they found that male represent $59.1 \%$ and female were $40.9 \%$.Others reported the same prevalence e.g Soliman et al., 2001, Shahnaz et al., 2004, Akira et al., 2005, Thomas et al., 2010, Emad et al., 2013 and Hsiu-Chi Hung et al., 2013.

In the current study more than three quarters of participants were illiterate or read and write. In agreement with our finding of Hadi et al (2009) they stated that more participants are illiterate or have primary school education .Other found lower percent of their patients have primary education ranged from 37.3\%(Ahmet et al., 2008) to 62\% (Donald et al., 2009, Thomas et al., 2010 and Salvador et al., 2011). Contrary to Shahnaz et al., (2004) reported that $41 \%$ of their participants had received a college education.

In the present study more than three quarters of the participants were married. This higher prevalence of marriage among colorectal cancer was reported by others. Maria et al., (2012) stated that the married participants represent $89.7 \%$ of their sample. Other studies revealed that participants were married in range of 58.3\%: $91.5 \%$ ( Hsiu-Chi Hung et al., 2013; Hadi et al., 2009; Ahmetet al, 2008; Salvador et al., 2011; Akira et al., 2005; Shahnaz et al., 2004; Donald et a.l, 2009 .It's clear that the late onset of the illness affected these around age of 45 years who expected of beings married at this age. In the current study $76.8 \%$ of participants were not working, similarly Volker et al., (2004) found that $78.4 \%$ of the participants are not working. In the 
current study there were $76.16 \%$ of participants live in rural area, Also Emad et al., (2013) found that most of the patients live in rural areas $(67.4 \%)$ and Soliman et al., (2001) stated that $78 \%$ live in rural area. In contrast both Darlene et al., (2012) and Maria et al., (2012) found that most participants live in urban area, as well as $80.8 \%$ of the participants in these study were in middle socioeconomic class, while Astrid et al., (2012) stated that $64.4 \%$ are in middle socioeconomic status.

Cancer colon is reported to be the site of prevalence. This result is in agreement with Akira et al., (2005), Astrid et al., (2012) David et al., (2013), and Emad et al., (2013) they reported that most of participants have cancer colon. The range of prevalence of cancer colon reported to be

$56 \%: 75.0 \%$ of colorectal cancer patients as reported by (Ahmed et al., 2002, Thomas et al., 2010 \& David et al., 2013). These figure are consistent with the finding of the present study, which showed more than half of participants have cancer colon. In the current study more than half of cancer colon patients have weight loss, slightly less than half have of patients have decrease appetite, less than quadrant of them have colostomy and slightly more than half have received nonspecific treatment .

Signs and symptoms of cancer colon are the same as cancer rectum and called together colorectal cancer. In which the patients complained of diarrhea or constipation, feeling that bowel does not empty completely, finding blood (either bright red or very dark) in stool, finding stools are narrower than usual, frequently having gas pains or cramps, or feeling full or bloated, losing weight with no known reason, feeling very tired all the time, and or having nausea or vomiting National Cancer Institute, (2006). In rectal cancer patients will complain from rectal bleeding, rectal itching, pain in the anal area, change in the diameter of stool, abnormal discharge from the anus, swollen lymph nodes in the anal or groin areas (American Cancer Society, 2013)

In the present study, there was no significant affection for QOL according demographic characteristics in colorectal cancer patients. Impaired QOL was common among females, married patients, patients live in rural area, illiterate or read and write, not working, less than usual sexual state, patients have weight loss, bowel habit change, decrease in appetite, moderate socioeconomic status and those with colostomy. Impaired of QOL is common in colorectal cancer patients as reported by Gray et al., (2011) \& Pan \& Tsai(2012) who stated that the presence of diarrhea, incontinence, fatigue and pain in addition to having direct effects on QOL influenced the daily activities and hobbies, and interfere with family and social life.
Volker et al., (2004) found that CRC patients scored their physical, role, cognitive, and global health functioning only as slightly worse which is similar to our patients and most of them showed moderate impairment. More severe limitations were observed in Volker et a.,l (2004) participant in the emotional and social functioning and fatigue, dyspnea, insomnia, constipation, diarrhea, and financial difficulties. Holzner et al., (2001); Ganz et al., (2002) \& Wei et al., (2002) stated that long-term effects of fatigue and sexual problems have been reported. It's clear that impairment of QOL in females may be related to their feeling of responsibility about their family, their children and sexual relation with partner. As well as effect of disease and side effects of treatment affect negatively of QOL of them.

According to SCL-90-R, the cutoff point of beings the patients need psychiatric assistance of any domain was $\geq 60$ in the present study colorectal cancer, have higher prevalence as regard somatization, hostility, depression, Anxiety and psychosis higher than in Egyptian general population, in mood disorders and anxiety disorders (Ghanem et al., 2009).

Also, many authors who studied these dimensions using different scales reported high prevalence than that was reported in the present study Fafouti et al., (2010) used SCL-90-R, TheMontgomery-Asberg Depression Rating Scale (MADRS) and the Spielberger State-Trait Anxiety Inventory (STAI) to assess depression, anxiety and found higher prevalence anxiety, depression, and general symptoms of psychopathology. Stefano et al., (2013)reported higher prevalence of distress, depression and anxiety in CRC patients than the general population. Also Thomas et al., (2010) found increased paranoid ideation, Psychoticism, interpersonal sensitivity, anxiety and depressive symptoms increased in CRC patients.

Nancy \& Donald, (2007) used the Centre for Epidemiological Studies-Depression scale (CES-D) and stated that over $30 \%$ of cancer patients were considered depressed, Maria et al., 2012 who used Epidemiologic Studies Depression Scale (CES-D) found that higher levels of depression were observed in cancer colon patients. Akira et al., 2005 who used the Hospital Anxiety and Depression Scale (HADS) found that $7.8 \%$ of participants have anxiety and $36.7 \%$ of participants have depression and Ahmet et al., (2008) who used Beck Depression Inventory (BDI) stated that $23.6 \%$ of the participants were depressed. Marrs, (2006)found that $30 \%$ of cancer patients suffer from chronic anxiety.

Marta et al., (2010) who used The Beck Depression Inventory (BDI) \& The State-Trait Anxiety Inventory (STAI) found that $31.6 \%$ of colorectal cancer patients 
have depression. It is clear that fear of death, loss of health commonly associated with somatic symptoms such as weight loss, fatigue, and motor difficulty. Intense feelings of tension, nervousness and worry, i.e. state anxiety, is an expected and well-known psychological response to a malignant disease and it is related to the emotional turmoil that follows diagnosis, anticipated prognosis, treatment, and its side-effects (Derogatis et al, 1983; Alexander et al., 1993; Hammer et al., 2009).

Hostility and paranoid ideation, anger, aggressiveness were found to be high in our patients. It's clear that these symptoms are not specific to certain type of tumor. This might be related to malignancy itself anywhere. As Fafouti et al., ( 2010)found their associated with breast cancer patients and stated that anger / aggressiveness were linked to each other and specifically they were suppressed feeling helplessness and repression seem to be two key factors closely associated and interrelated with anger/aggressiveness, as repressed hostility, negative emotions and a feeling of loss of control often described by malignancy patients are likely to contribute through a vicious circle to unfavorable prognosis, while denial/minimizing have been reported to be associated with a more favorable prognosis (Iwamitsu et al., 2005)

Females significantly at higher risk than males to develop anxiety and hostility. Also, they tend to develop somatization, interpersonal sensitivity, depression, phobia, paranoia and psychosis higher than males. Other studies reported same finding. Landsbergen et al., (2011) stated that female patients reported higher levels of psychological distress than male patients, Akira et al., (2005) found that higher rates of depression had been described among women, but anxiety was similar in both genders.

Contrary to this, Thomas et al., 2010 found that men were at greater risk for further developing depressive, it's may due to that woman were the care giver for their family and this lead worrying about cancer diagnosis and fear on their family if they died. Maria et al., (2012) stated that depression also affects men and women with cancer equally and also stated that there was not statistically difference between depression and gender as well as between depression and age. This is similar to present study. In the current study more than two third of patients with moderate quality life have somatization, two third of patient with low quality of life have obsession .slightly less than two third of patients with moderate quality have depression and anxiety. While Akira et al., (2005) found depression more highly correlated than anxiety with reduced QL. Most of patient of moderate quality of life have hostility and phobia.

Line of treatments might be associated with high prevalence different dimension of SCL-90-R. More than half of patients receive chemotherapy complain of interpersonal sensitivity and hostility, also about one third of them have phobia and obsession in addition to that that less than one quarter complain from psychosis, depression, paranoia and anxiety. This agree with Pandey et al., (2006) found depression to be present in less than one quarter in cancer patients undergoing chemotherapy. Marta et al., (2010) found that third of the participants who receive chemotherapy have anxiety more than other items of SCL .

In the present study patients came for follow up and received nonspecific treatment for gastric upset, diarrhea or general support complain more of different dimensions of SCL- 90-R. This might be due to chronicity of illness, uncertainty about their future, affection of their QOL, frequent follow up visit to the hospital or investigation provoked. Their prior experience of diagnosis of cancer associated with the suspicious of recurrence and threaten effect on their life.

Patients with colostomy either permanent or temporary was complained of paranoia as those patients tend to be isolated,withdrawn from social activity because suspicious about looking of others at them and others thought that cancer is a infectious disease, bad odor of colostomy and body image after colostomy. In these study $30.8 \%$ of patients have depression and $21.4 \%$ of them have anxiety partially agree with White, (1997) who stated that $25 \%$ of people who have stoma operations experience severe problems with anxiety, depression and other negative emotions at some stage during the years after stoma surgery.

\section{Conclusions}

- Based on the results of the present study, it can be concluded that, high percentage of colorectal cancer patients were females, more than two thirds of them were living in rural area, illiterate or can read and write, not working, married and in the middle socioeconomic level, high percentage of patients had a moderate quality of life and most of them have cancer colon, less than half of the patients have loss of appetite, more than half of them have weight loss. Colostomy and chemotherapy represent part of their lines of treatment.

- As regard SCL-90-R there were number of patients have somatization, hostility, anxiety, 
depression, phobia, paranoia, psychosis, interpersonal sensitivity and obsession.

- Patients with cancer rectum were at high risk to developing interpersonal sensitivity or psychosis $(\mathrm{p}=0.02,0.01$ respectively), female patients were at high risk for developing anxiety or hostility ( $\mathrm{p}=0.01,0.02$ respectively), patients with moderate quality of life were at high risk for developing different dimensions of SCL-90-R items $(\mathrm{p}=0.00)$, patient receive nonspecific treatment at high risk for developing somatization, depression or anxiety $(\mathrm{p}=0.00$, $0.02, \quad 0.000$ respectively), patients with colostomy are at high risk to developing paranoia $(\mathrm{p}=0.00)$, single patients were at high risk to develop obsession $(\mathrm{p}=0.00)$ and not worked patients are at high risk to developing obsession, interpersonal sensitivity, hostility or psychosis $(\mathrm{p}=.000, .010, .050 \& .010$ respectively).

\section{Recommendations}

Based on the findings of the present study, the following recommendations are suggested:

1- Further research specified to study risk factor associated with the development of psychotic symptoms.

2- Educational program should be planned to educate nursing staff about stressor related to colorectal cancer

3- Liaison psychiatric nurse should be available to deal with patients problems in colorectal cancer patients.

\section{References}

1. Abad EIWahab E., Ahmed M., Adly S., Anter A., (2002): A study of the impact of work on quality of life among psychiatric patients, master thesis in Psychiatric Nursing Assiut University, No103.

2. Ahmed N., Niu J., Dorahy D., Gu X., Andrews S., Meldrum C., Scott R., Baker M., Macreadie I., Agrez M., (2002): Direct ERKintegrin binding: implications for tumor growth. Oncogene.;21:1370-1380.

3. Ahmet, A., \& Omer B., Ozkan G., Ilhan O., Meliha D., Ugur Y., (2008): Quality of life, anxiety, \& depression in Turkish colorectal cancer patients. Support Care Cancer. Apr; vol 18(4):417-21. doi: 10.1007/s00520-009-0679-2.

4. Akira T., Kentaro N., Kenshi H., Naokuni Y., Miki S., \& Mitsuo K., (2005): Anxiety, depression and quality of life in colorectal cancer patients. Int J Clin Oncol. 2005 Dec; vol 10(6):411-7
5. Almanza, M., Holland, C., (2000): Psicooncología: estado actually perspectives futures. Rev Inst Nal Cancerol;46:196-206.

6. Alexander P., Dinesh N., \& Vidyasagar M., (1993): Psychiatric morbidity among cancer patients and its relationship with awareness of illness and expectations about treatment outcome Acta Oncol 32: 623-62.

7. American cancer society (2013): Cancer Facts and Figures Inc. No. 500813

8. Ashbury F., Madlensky L., Raich P., Thompson M., Whitney G., Hotz K, Kralj B., Edell W., (2003): Antidepressant prescribing in community cancer cre. Support Care Cancer. 11: 278-285.

9. Astrid, A., Kari, Fish, Christina A., Clarke,Dee W., West, Scarlett L., Gomez, Iona Cheng (2012): Examining the Association Between Socioeconomic Status and Invasive Colorectal Cancer Incidence and Mortality in California. Cancer EpiSSSdemiol Biomarkers Prev. 2012 Oct; 21(10):1814-22.

10. Center M., Jemal A., Ward E., (2009): International trends in colorectal cancer incidence rates. Cancer Epidemiol Biomarkers, 18, 1688-94.

11. Darlene V., Amr, S., Soliman S., Gilbert S., Blachley, Ahmed H., Mohamed R., Laura S., Rozek, Ibrahim A., Seifeldin (2012): Age distribution, polyps \& rectal cancer in the Egyptian population-based cancer registry. World Journal Gastroenterology, Agu 14, 18(30): 3997-4003.

12. David Goldsbury, Mark Fort Harris, Shane Pascoe, Ian Olver, Michael Barton, Allan Spigelman, Dianne O., Connell (2013): Socio demographic and other patient characteristics associated with time between colonoscopy and surgery, and choice of treatment centre for colorectal cancer: a retrospective cohort study. BMJ Open ;2:e001070.

13. Demetri, G., (2001): Anemia \& its functional consequences in cancer patients: current challenges in management and prospects for improving therapy. British Journal Cancer 84 (1), 17023.

14. Derogatis, L., Lipman, R., \& Covi, L., (1973): The SCL-90, an outpatient psychiatric rating scale Preliminary report. Psychopharmacology Bulletin Journal; vol 9(1):13-28

15. Derogatis, L., Rickels, K., \& Rock, A., (1976): The SCL-90 \& the MMPI, A., step in the validation of a new self-report scale, British Journal of Psychiatry; vol 128(5):280-289.

16. Derogatis L., Morrow G., Fetting J., Penman D, Piasetsky S، Schmale A., Henrichs $M$ and 
Carnicke J., (1983): The prevalence of psychiatric disorders among cancer patients. JAMA 249: 751-757.

17. Die ,T.,( 2007) Impacto psicosocial del cáncer colorrectal. Psicooncología. Rev SEOM;12:19. 21

18. Dietrich, J., Han, R., Yang, Y., MayerProschel, M., \& Noble, M., (2006): "CNS Progenitor Cells and Oligodendrocytes Are Targets of Chemotherapeutic Agents in Vitro and in Vivo," Journal of Biology, 5.

19. Donald M., Sharp, Mary B., Walker, Julie S., Bateman, Fiona Braid, Claire Hebble white, Teresa Hope, Michael Lines, Andrew A., Walker \& Leslie G., Walker (2009): Demographic characteristics of patients using a fully integrated psychosocial support service for cancer patients. BMC Research Notes, 2:253.

20. Emad H., Sherif F., Soliman E., Kammash M., and Mohammed Faisal Msc, (2013): Assessment of quality of life of colorectal carcinoma patients after surgery. World Journal of Colorectal Surgery Volume 3, Issue1, article 4.

21. Fafouti,T., Paparrigopoulos , Y., Zervas, A., Rabavilas , N., Malamos 2 , 1 Liappas \& C., Tzavara (2010): Depression, Anxiety \& General Psychopathology in Breast Cancer Patients: A Cross-sectional Control Study.in vivo International Journal of Experimental and Clinical Pathophysiology and Drug Research 24: 803-810.

22. Fillipovic B., Fillipovic B., kerkez M., Millnic N., Randelovic T., (2007): Depression $\&$ anxiety levels in therapy -nive patients the inflammatory bowel disease and cancer of the colon. World J Gastroenterology; 13(3): 438 443.

23. Ganz, K., Rimer, K., \& Lewis, F., (2002): Health behavior \& health education ( $3^{\text {rd }}$ ed.). San Francisco, CA:Jossey-Bass. Gastroenterol; 13(3): 438-443.

24. Gray N., Hall S., Browne S., Macleod U., Mitchell E., Lee A., Johnston M., Wyke S., Samuel L., Weller D., et al (2011): Modifiable $\&$ fixed factors predicting quality of life in people with colorectal cancer. British journal of cancer, 104(11):1697-1703.

25. Ghanem, M., Gadallah, M., Meky, F., Mourad S., \& El-Kholy G., (2009): National Survey of Prevalence of Mental Disorders in Egypt: preliminary survey.East Mediterr Health J., JanFeb;15(1):65-75.

26. Hadi, R., Asadollahi, A., Talei (2009): Anxiety, Depression \& Anger in Breast Cancer Patients Compared with the General Population in Shiraz,
Southern Iran.Iranian Red Crescent Medical Journal11(3):312:317.

27. Hammer M., Chida Y., \& Molloy G., (2009): Psychological distress and cancer mortality. J., Psychosom Res 66: 255-258.

28. Holzner, B., Kemmler, G., Kopp, M., Moschen, R., Schweigkofler, H., Dunser, M., SpernerUnterweger, B., (2001): Quality of life in breast cancer patients -not enough attention for long-term survivors. Psychosomatics, 42,117-123.

29. Hsiu-Chi Hung1, Tsui-Wei Chien, ShiowLuan Tsay, Hewi-Ming Han, Shu-Yuan Liang (2013): Patient \& Clinical Variables Account for Changes in Healthrelated Quality of Life and Symptom Burden as Treatment Outcomes in Colorectal Cancer: A Longitudinal Study.

30. http://seer.cancer.gov/csr/1975_2002:/results_ merged/topic_age_dist.pdf.

31. Hurter, B., Bush, N., (2007): Cancer-related anemia: clinical review and management update. Clinical Journal Oncology Nursing 11, 349e359.

32. Hyodo I., Suzuki H., Takahashi K., (2010): Present status \& perspectives of colorectal cancer in Asia: Colorectal Cancer Working Group Report in 30th Asia-Pacific Cancer Conference. Jpn J Clin Oncol, 40, 38-43.

33. Iwamitsu Y., Shimoda K., Abe H., Tani T., Okawa M., \& Buck R., (2005): The relation between negative emotional suppression and emotional distress in breast cancer diagnosis and treatment, Anxiety emotional suppression, and psychological distress before and after breast cancer diagnosis. Psychosomatics. 18: 201-215.

34. Jadoon N., Munir W., Shahzad M., Choudhy Z., (2010): Assessment of depression \& anxiety in adult cancer outpatients : a cross-sectional study. B., Cancer ; 10:594.

35. Jemal A., Siegel R., Ward E., et al (2008): Cancer statistics, 2008. CA Cancer J Clin; 58:7196.

36. Landsbergen K., Prins, H., Brunner, N., Hoogerbrugge (2011): Shortened time interval between colorectal cancer diagnosis. am Cancer. Mar 2011; 10(1): 51-57.

37. Lehman A., Possidente S., \& Hawker, F., (1986): The quality of life of chronic patients in a state hospital \& in community residence .Hospital and Community Psychiatry, 37:901907 .

38. Maria, L., George, B., Aikaterini, F., Fratzana, Sofia, Z., (2012): Evaluation of depression in colon cancer patients.

39. Marrs J., (2006): Stress, fears and phobias: the impact of anxiety. Clin J Oncology Nurse. Jun; 10(3):319-22. 
40. Marta, M., Celina, T., Fujiyama, O., \& M., Manoukian, (2010): Depression and Anxiety in Colorectal Cancer Patients. Journal of Gastrointestinal Cancer September, Volume 41, Issue 3, pp 179-184.

41. Maté M., Gil M., Lluch S., (2003): Quimioterapia, hormonoterapia einmunoterapia. In: Die T., ed. Psicooncología. España: ADES;283:289.

42. Mystakidou K., Tsilika E., Parpa E., Katsouda E., Galanos A., Vlahos L., (2005): Assessment of anxiety \& depression in advanced cancer patients and their relationship with quality of life. Qual Life Res. (2005): 14:1825 -33.

43. Nancy Humpel \& Donald C., Iverson (2007): Depression \& quality of life in cancer survivors: is there a relationship with physical activity. The International Journal of Behavioral Nutrition and Physical Activity 4:65.

44. National Cancer Institute, (2006): what you need to know about cancer of the colon and rectum, NIH publication number 06-1552.

45. National Cancer Registry(2006): Ministry of Health Malaysia. Malaysian Cancer Statistics Data And Figure Peninsular Malaysia .

46. Northern Ireland Cancer Registry, (2008): Online cancer statistics. Available from http://www.qub.ac.uk/research centers/nicr/Data/Online Statistics.

47. Office for National Statistics ONS, (2008):Cancer statistics registrations. Registrations of cancer diagnosed in 2005 England. Available From http://www.statistics.gov.uk/downloads/theme_h ealth/MB1_36/MB1_No36_2005.pdf on Work, Social Networks, and Health Care Response,"

48. Pan L., Tsai Y., (2012): Quality of life in colorectal cancer patients with diarrhoea after surgery: a longitudinal study. Journal of clinical nursing, 21(156):2357-2366.

49. Pandey, M., Sarita, G., Devi, N., Thomas, B., Hussain, B., Krishnam, R., (2006): Distress, anxiety, and depression in cancer patients undergoing chemotherapy. World Journal of Surgical Oncology 4, 68.

50. Pollack, J., Holm, T., Cedermark, B., Attman, D., Holmstroim, B., Glimelious, B., Melgree, A., (2006): Late adverse effects of short-course preoperative radiotherapy in rectal cancer. British Journal of Surgery 93 (12), 1519e1525.

51. Salvador A, Hilda G ., Aura L., Germán C., Luis F., (2011): Psychosocial adjustment in colorectal cancer patients undergoing chemotherapy or chemo radiotherapy. Cir Cir. Sep-Oct;79(5):439-46.
52. Santos, Z., (1995): Reacções emocionais e apoio psicológico ao doente com doença grave. Cuidar $2,3 \mathrm{e} 7$.

53. Schag C., Ganz P., Wing D., (1994): Quality of life in adult survivors of lung, colon and prostate cancer. Qual Life Res 3:127-141.

54. Schmidt, C., Bestmann, B., Kuchler, T., Longo, W., Kremer, B., (2005): Ten year historic cohort of quality of life and sexuality in patients with rectal cancer. Diseases of Colon and Rectum 48 (3), 483e492.

55. Shahnaz Sultan M., Deborah A., FisherM., Corrine I., Voils Ph., Anita Y., Kinney, Ph., Robert S., Sandler M., Dawn ProvenzaleM., (2004): Impact of functional support on healthrelated quality of life in patients with colorectal cancerWiley online library Volume 101, Issue 12, Article first published online: $9 \mathrm{NOV}$.

56. Soliman A., ML Bondy, S., El-Badawy, N., Mokhtar, S., Eissa , S., Bayoumy, I., Seifeldin, P., Houlihan , J., Lukish , T., Watanabe, A., On On Chan, D., Zhu, C., Amosn, B., Levin and S., Hamilton (2001): Contrasting molecular pathology of colorectal carcinoma in Egyptian \& Western patients.

57. Sprangers M., Taal B., Aaronson N., (1995): Quality of life in colorectal cancer. Stoma vs. non stoma patients Dis Colon Rectum Apr; 38(4):361-9.

58. Sprangers, M., de Velde A., Aaronson N., Taal B., (1993) Quality of life following surgery for colorectal cancer. A., literature review. Psychooncology 2:247-259.

59. Stefano,M., Marria J., F., Giuseppe G., Antonio M., Gabriele G., Alessio P., Santi G., Francesco B., \& Antonio B., (2013): Health related quality of life in colorectal cancer patients: state of the art.BMC Surgery, 13 (Suppl 2):S15

60. Thomas H., Vassiliki P., Augoustina A., Eleftherios O., ,Kampletsas, Nicholas P., (2010): Personality variables as predictors of early non-metastatic colorectal cancer patients' psychological distress \& health-related quality of life : A one-year prospective study. Journal of Psychosomatic ResearchVolume 70, Issue 5, Pages 411-42.

61. Urquidi T., Montiel C., Gálvez L., (1999): Ajuste psicosocial y afrontamiento en pacientes con cáncer de mama. Rev Sonorense Psicol ;13:30-36.

62. Volker A., Henrike M., Christa S., Hartwig Z., \& Hermann Brenner (2004): Quality of Life in Patients With Colorectal Cancer 1 Year After Diagnosis Compared With the General 
Population: A., Population-Based Study. Journal of clinical oncology volume 22, number 23.

63. Wei J., Dunn R., Sandler H., McLaughlin P., Montie J., Litwin M., (2002): Comprehensive comparison of health-related quality of life after contemporary therapies for localized prostate cancer. J Clin Oncol ; 20: 557-566.

64. Welsh Cancer Intelligence \& Surveillance Unit WCIS (2008): Cancer Incidence in Wales ( 2002-2006). Available from http://www.wales .nhs.uk/sites3/page .cfm?orgid ,242\&pid,30138.

65. White C., (1997): living with a stoma: a practical guide to coping with colostomy, ileostomy or urostomy. Sheldon Press, London. 